\title{
Conversion of Skeletal Society of Radiology annual meeting abstracts to publications in 2010-2015
}

\author{
Daniel Tritz ${ }^{\text {Corresp.. }}{ }^{1}$, Leomar Bautista ${ }^{2}$, Jared Scott ${ }^{1}$, Matt Vassar ${ }^{3}$ \\ 1 Oklahoma State University Center for Health Sciences, Tulsa, United States \\ 2 Department of Radiology, Oklahoma State University Medical Center, Tulsa, United States \\ 3 Department of Psychiatry, Oklahoma State University Center for Health Sciences, Tulsa, United States \\ Corresponding Author: Daniel Tritz \\ Email address: daniel.tritz@okstate.edu
}

Background: Material presented at conferences is meant to provide exposure to ongoing research that could affect medical decision making based on future outcomes. It is important then to evaluate the rates of publication from conference presentations as a measure of academic quality as such research has undergone peer review and journal acceptance. The purpose of this study is to evaluate the fate of abstracts presented at the Skeletal Society of Radiology Annual Meetings from 2010-2015.

Materials and Methods: Conference abstracts were searched using Google, Google Scholar, and PubMed (which includes Medline) to locate the corresponding published reports. The data recorded for published studies included date published online, in print, or both; the journal in which it was published; and the 5year journal impact factor. When an abstract was not confirmed as published, authors were contacted by email to verify its publication status or, if not published, the reason for nonpublication.

Results: A total of 162 abstracts were published out of 320 presented (50.6\%) at the SSR conferences from 2010 to 2015 with 59.9\% (85/142) of publications occurring within two years of the conference date (not counting abstracts published prior to conference). Mean time to publication was 19 months and is calculated by excluding the 20 (12.3\%) abstracts that were published prior to the conference date. The median time to publication is 13 months (25th - 75th percentile: $6.25-21.75$ ). The top two journals publishing research studies from this conference were Skeletal Radiology and The American Journal of Roentgenology. These journals accepted 72 of the $162(44.4 \%)$ studies for publication. Of the 14 authors who responded with 17 reasons for not publishing, the most common reasons were lack of time (7 $41.2 \%$ ), results not important enough (4 - 23.5\%), publication not an aim (3 - 17.6\%), and lack of resources (3 - 17.6\%).

Discussion: At least half of the abstracts presented at the annual meeting for the Society of Skeletal Radiology are accepted for publication in a peer-reviewed journal. The majority (59.9\%) of these publications were achieved within two years of the conference presentation. The rate at which presentations are published and the journals that accept the abstracts can potentially be used to compare the importance and quality of information at conferences. 
1 Title: Conversion of Skeletal Society of Radiology Annual Meeting Abstracts to Publications in $2 \quad 2010-2015$

3

4 Authors: Daniel Tritz; Leomar Bautista, DO; Jared Scott; Matt Vassar, PhD.

5

6 Affiliations: Oklahoma State University, Tulsa, OK: Center for Health Sciences, (Tritz and

7 Scott); Department of Radiology, Oklahoma State University Medical Center (Bautista);

8 Department of Psychiatry, Oklahoma State University Center for Health Science (Vassar).

9

10 Corresponding Author: Daniel Tritz

11 Email: Daniel.tritz@okstate.edu

12

13

14

15

16 


\section{Abstract}

Background: Material presented at conferences is meant to provide exposure to ongoing research that could affect medical decision making based on future outcomes. It is important then to evaluate the rates of publication from conference presentations as a measure of academic quality as such research has undergone peer review and journal acceptance. The purpose of this study is to evaluate the fate of abstracts presented at the Skeletal Society of Radiology Annual Meetings from 2010-2015.

24

Materials and Methods: Conference abstracts were searched using Google, Google Scholar, and PubMed (which includes Medline) to locate the corresponding published reports. The data recorded for published studies included date published online, in print, or both; the journal in which it was published; and the 5-year journal impact factor. When an abstract was not confirmed as published, authors were contacted by email to verify its publication status or, if not published, the reason for nonpublication.

Results: A total of 162 abstracts were published out of 320 presented (50.6\%) at the SSR conferences from 2010 to 2015 with $59.9 \%$ (85/142) of publications occurring within two years of the conference date (not counting abstracts published prior to conference). Mean time to publication was 19 months and is calculated by excluding the $20(12.3 \%)$ abstracts that were published prior to the conference date. The median time to publication is 13 months (25th 75th percentile: 6.25 - 21.75). The top two journals publishing research studies from this conference were Skeletal Radiology and The American Journal of Roentgenology. These journals accepted 72 of the $162(44.4 \%)$ studies for publication. Of the 14 authors who responded with 17 reasons for not publishing, the most common reasons were lack of time (7 $41.2 \%$ ), results not important enough (4 - 23.5\%), publication not an aim (3 - 17.6\%), and lack of resources $(3-17.6 \%)$. 
43

44 Discussion: At least half of the abstracts presented at the annual meeting for the Society of

45 Skeletal Radiology are accepted for publication in a peer-reviewed journal. The majority (59.9\%)

46 of these publications were achieved within two years of the conference presentation. The rate at

47 which presentations are published and the journals that accept the abstracts can potentially be

48 used to compare the importance and quality of information at conferences.

49

50

51

52

53 


\section{Introduction}

55 A 2010 study on the global burden of disease found that the prevalence of musculoskeletal

56 disorders was $8.4 \%$; however, this rate increased to $20-40 \%$ in people over the age of 80 years

57 (Smith et al., 2014). To improve outcomes for patients with these disorders, scientific

58

59

60

61

62 advancements are needed. The dissemination of scientific knowledge occurs through periodic meetings as well as through the publication of research in peer-reviewed journals. The Skeletal Society of Radiology (SSR) works to develop skills and conduct research in musculoskeletal radiology. This society holds an annual meeting to encourage research activity and dissemination, stimulate collaboration among its members, and refine subspecialty expertise through continuing medical education and presentation opportunities. The research abstracts submitted to this meeting undergo formal peer-review and while beneficial, evidence suggests that publication rates for conference abstracts are higher for those with positive findings, randomized designs, and larger sample sizes (Langenberg \& von Elm 2007). These studies have also found that oral presentations and those falling into the category of experimental research are also more frequently published (Easterbrook et al., 1991; Dickersin, Min \& Meinert, 1992; Scherer, Langenberg \& von Elm, 2007).

In this study, we evaluate the fate of abstracts presented at the SSR (Skeletal Society of Radiology) Annual Meeting from 2010 to 2015. We also analyze the length of time for abstracts to be published from the date of the conference and catalogued the top ten most common journals that publish these studies after presentation. In addition, we explore the reasons for nonpublication to evaluate the most common barriers. To our knowledge, this is the first study examining the publication rates from a radiology or musculoskeletal conference within the United States or for this society's annual meeting. 
80

81

82

83

84

85

86

87

\section{Methods}

\section{Oversight and Reporting}

This study did not meet the regulatory definition of human subjects research as defined in 45 CFR 46.102(d) and (f) of the Department of Health and Human Services' Code of Federal Regulations and, therefore, was not subject to Institutional Review Board oversight. We applied relevant Statistical Analyses and Methods in the Published Literature (SAMPL) guidelines for reporting descriptive statistics. We developed our methodology by consulting previous studies on the publication rates of conference abstracts (Hoag, Elterman \& Macneily, 2006).

\section{Locating Conference Abstracts}

SSR oral and poster presentation abstracts from 2010 to 2015 were located using information from the SSR website about past conference summaries on PubMed. This time period was selected based on The European Society of Skeletal Radiology's decision in 2011 that a duration of two years from research conception to publication is acceptable (Parkar, Vanhoenacker \& Adriaensen van Roij, 2013). Including abstracts from 2010 to 2015 leaves at least two years for any abstracts to reach publication. The search period for the abstracts included the period 2009-2017 to allow for any presentations published before the first conference in 2010 and two years to submit projects after the final conference in 2015.

\section{Search Strategy for Published Manuscripts of Conference Abstracts}

We attempted to locate the published reports of conference abstracts using a predefined search algorithm (Figure 1). The search algorithm was developed by two investigators and pilot-tested on 25 abstracts. We assessed the optimal order in which to search databases as Google, Google Scholar, and PubMed. We also varied the searches using combinations of keywords and authors' names as well as full title searches to determine which strategy would provide the greatest precision to locate published reports. One investigator first searched the three databases (in the order: Google, Google Scholar, and PubMed) by using the full conference abstract title. If this strategy failed to locate a published report, then the investigator performed 
106 the search using the first author's last name and keywords from the abstract. For those

107 abstracts in which no published report could be located, a second investigator repeated the

108 search strategy. These searches were made in June/July of 2017.

109 Contacting Authors

110 If no publication had been located after two searches, a standardized email was then sent to the

111 first author of the conference abstract. If contact information for this author could not be located,

112 we then contacted all authors for whom an email address was found (Figure 1). This email

113 provided authors with the opportunity to comment on whether the study was published and, if

114 so, the reference for the publication. In the event of non-publication, abstract authors were

115 asked to provide a reason for non-publication. The standardized response options for non-

116 publication were based on a systematic review by Song et al. that analyzed 38 survey reports

117 on investigator-reported reasons for non-publication (Song, Loke \& Hooper, 2014).

118 Figure 2 provides a step-wise description of the process later discussed in the results. The initial

119 number of papers identified was from the investigators' data collection before contacting the

120 listed authors. Email responses from the authors were either the reasoning why the abstract

121 was not published or a link to the article that was not found previously. The newly located

122 articles are added into the total at the bottom of the figure and the end result.

\section{Data Collection}

124 After locating a published study thought to be a conference abstract, we then compared the 125 author list, methods, and results. If at least two of the following criteria were met, we counted 126 the abstract as published: 1) the results from the published report matched the results in the 127 conference abstract, 2) the methodology from the published report was similar to the 128 methodology described in the conference abstract, or 3) the first author of the conference 129 abstract was included in the author list of the published study.

130 Data were extracted from the published study using a standardized, pre-specified, and piloted

131 Google Form. We extracted the following information when available: publication title, institution 
132 of first author, date submitted to journal, date accepted for publication, date of in-print

133 publication, date of online publication, journal name, and 5-year journal impact factor. We

134 calculated the time to publication based on the number of months between the date of the

135 conference where presented and the publication date online due to the lack of data regarding

136 date accepted for publication. When necessary, we used a zero number of months to indicate

137 studies in which publication occurred prior to conference commencement. The 5-year journal

138 impact factor was located by searching the article name in the "Web of Science" from clarivate 139 analytics.

140

141

142

143 


\section{Results}

145

146 From 2010 to 2015,320 abstracts were presented. Of these, 162 (50.6\%) were found to be

147 published in a peer-reviewed journal (Figure 2). The highest rate of publication by the time of

148 data extraction was in 2013 at $63.6 \%$ (28/44) and the lowest was the same in 2012 and 2015 at

$14945.1 \%$ (32/71 and 23/51, respectively) (Figure 3). Throughout the time period, publication times

150 ranged from 0 (prior to the presentation) to 77 months after the conference date with a median

151 of 13 months (25th - 75th percentile: 6.25 - 21.75). Of the 162 published articles, $20(12.3 \%)$

152 abstracts were published prior to the conference date when it was presented and are removed

153 from calculations for times to publication. 19 months with $85(59.9 \%)$ of the 142 presentations

154 accepted for publication within two years after presentation. The two years broken down

155 includes $53(32.7 \%)$ within the first year and $52(32.1 \%)$ within the second. A Kaplan-Meier

156 analysis was done to show the proportion of the abstracts which were published measured in

157 months after presentation at the conference (Figure 4). This analysis includes abstracts that

158 were eventually published and eliminates values of 0 (published prior to conference).

159

Abstracts from the SSR conference were published in 47 journals. There were 12 journals that accepted three or more abstract presentations for full-text publication (Figure 5). The top two journals that published abstracts from this conference were Skeletal Radiology and The American Journal of Roentgenology. Skeletal Radiology published 37 of the total $162(22.8 \%)$ and The American Journal of Roentgenology accepted 35 of 162 (21.6\%).

165

166

167

168

169

170

Of the 320 abstracts, 161 were not found to be published as full-text articles. Email addresses were found for 153 out of 161 presentations. Authors associated with multiple projects were sent one email. A total of 110 unique emails were sent out with 22 being returned as invalid or out-of-date addresses. Seventeen responses were recorded while 114 authors for presentations did not reply. Three responses indicated that the study was published with authorship 
171 information and 14 confirmed the paper was not published with 17 reasons why (Figure 2). The

172 most common barriers to publication were lack of time ( $n=7-41.2 \%)$, results not important

173 enough ( $n=4-23.5 \%)$, publication not an aim $(n=3-17.6 \%)$, and lack of resources $(n=3-$

$174 \quad 17.6 \%)$.

175 


\section{Discussion}

177

178 The publication rate of SSR conference abstracts from 2010 to 2015 was $50.6 \%$. Scherer et al.

179 evaluated 79 biomedical conferences and reported an overall publication rate of $44.5 \%$, which is

180 lower than the SSR. Because this is the first study of publication rates for a musculoskeletal

181 radiology conference in the United States, comparison can only be made with international

182 meetings. The rate of publication from the SSR conference is favorable compared with that of

183 the Canadian Association of Radiologists for 2005-2011 (28\%) (Dressler \& Leswick, 2015), the

184 Korean Radiological Society for 2001-2002 (23.6\%) (Ha et al., 2008), and the European Society

185 of Skeletal Radiology for 2008-2009 (45\%) (Parkar, Vanhoenacker \& Adriaensen van Roij,

186 2013). Miguel-Dasit et al. (2006a; 2006b) analyzed abstracts submitted to the European

187 Congress of Radiology (ECR). In 2000, abstracts submitted from United States investigators

188 were more likely to reach publication (62\%). This finding was confirmed when extending the

189 conference dates to include 2000-2004 (76\%). Loughborough et al. found that publication rates

190 for abstracts in the musculoskeletal category of this Congress were $51 \%$, which is very similar to 191 our findings (Loughborough et al., 2016).

192

193 Forty-seven journals published 162 abstracts presented at the 2010-2015 SSR conferences.

194 The two most common journals for publication were The Journal of Skeletal Radiology and The

195 American Journal of Roentgenology with 37 and 35 abstracts accepted, respectively. The

196 Journal of Skeletal Radiology has a 5-year impact factor of 1.913 and is the official journal of the

197 Society of Skeletal Radiology. The American Journal of Roentgenology has a higher 5-year

198 impact factor of 3.105 but is not associated with the SSR. The 5-year impact factor of journals

199 that published three or more articles ranges from 0 to 10.700 . Rodriques et al. found that higher

200 impact factors correlate with studies that use the highest level of evidence to support their 
201 outcomes (Rodrigues et al., 2014). In addition, the impact factor of a journal has been shown to

202 strongly correlate with the newsworthiness and methodological quality of its recent

203 publications(Lokker et al., 2012). Although the impact factor of a journal is useful as a binary

204 measurement for comparison in this study, there are many limitations for using it. A journals

205 impact factor can be influenced by a few recent papers being cited many times compared with

206 other publications. This imbalance makes the impact factor a better marker for comparing

207 journals with each other as opposed to individual papers due to varying numbers of citations per

208 paper (Adler et al. 2008).

209

210 The importance of journal impact factors for conferences corresponds to the quality of the

211 presented abstract. Abstracts for the SSR are reviewed and officially accepted by their

212 programming committee, which consists of the President-Elect as Chair, the SSR Secretary,

213 and one Society full member in good standing. There is little information on the criteria used to

214 select presentations other than a focus on correlating abstracts with session topics or program

215 needs and objectives. Blackburn et al. had reviewers use a five-point scale for the overall poster

216 as well as an evaluation of its importance, quality, implications for theory, and implications for

217 practice. This study found that based on the mean scores given for each presentation, $17-20 \%$

218 of subjective decisions for acceptance or rejection would have been reversed (Blackburn \&

219 Hakel, 2006). Kuczmarski et al. surveyed 27 large scientific conferences to evaluate the

220 screening process for conference abstracts to be presented and found that only $2(7 \%)$ made

221 the scoring process available to submitters and the public (Kuczmarski, Raja \& Pallin, 2015). As

222 a follow-up to the survey, they created a comprehensive scoring system that emphasized

223 transparency and objectivity. This scoring system asks reviewers to provide 0 to 2 points for

224 each of the seven categories that are outlined by general criteria for each grade, as well as

225 specific examples. We suggest that the SSR takes steps to ensure transparency in their scoring

226 process that may result in improved abstract quality (Kuczmarski, Raja \& Pallin, 2015). 
228 The most common reasons that authors cited for nonpublication were lack of time, results not important enough, publication not an aim, and lack of resources. Krzyzanowska et al. found lack of time to be a major barrier to publication in large randomized trials that were presented at the American Society of Clinical Oncology (Chapman et al., 2012). The belief that results were not important enough corresponds with the notion that publication is more likely when results are positive or when they correlate with a significant outcome (Hopewell et al., 2009). This phenomenon is known as "publication bias" and is a common problem in medical literature. Evidence suggests that editors are less likely to publish negative results, peer-reviewers are less likely to recommend them for publication and researchers are less likely to submit them in the first place (Dickersin, Min \& Meinert, 1992). This form of bias contributes to overestimated treatment effects because only statistically significant outcomes and large magnitude effect sizes are available to represent an intervention's effectiveness. The null or negative findings from studies of these same interventions are neither available nor known to the research community as they have not been published. Efforts to minimize publication bias have included alternative peer review models and negative results sections in journals. Such efforts will

243 hopefully contribute to positive changes on this important issue.

This study utilized multiple methods to identify publications from conference abstracts. The searches were made using Google Scholar, PubMed, and Google. These search engines could potentially miss articles published in databases that are not connected. An attempt was made to contact at least one author associated with each presentation through email. Although not all authors replied, the two-researcher search method and attempted contact with authors makes the potential for omissions limited. The conference summaries listed oral presentations for each abstract submitted to the annual conference, but poster presentation information was only listed

252 for two years. During these two years the rate of publication was similar to the overall 
253 publication rate; therefore, it was decided to not separate the data just for the two years of 254 posters. Finally, we did not impose time limits in our investigation; abstracts presented in 2010, 255 for example, would have a longer time window to reach publication than abstracts presented at 256 the 2015 conference. Given the Kaplan-Meier analysis (Figure 4) demonstrating the majority of 257 publications occurring within 2 years, it is likely that a majority of abstracts presented in later 258 years such as 2015 had reached their final fate by our study's end point.

259

260

261

262

263 


\section{Conclusion}

265 In summary, approximately half of the abstracts presented at SSR Annual Meetings are

266 eventually published. The rate of publication for abstracts presented at the SSR are higher than

267 those analyzed from radiology conferences in other countries like Canada and Korea, and are 268 comparable with the European College of Radiology. The majority of abstracts were accepted for publication by two years after the conference date. Those abstracts that do not reach publication are the result of a lack of time or resources, a perception of unimportant findings, and publication

271 not being an aim of the abstract author. Such reasons are problematic when they contribute to

272 publication bias and efforts should be made to encourage the publication of results, regardless of

273 the strength or direction of study findings. Furthermore, efforts should be made to increase the

274 transparency of the scoring rubrics used by conferences to increase the quality of the submissions 275 they receive. 


\section{References}

280 Adler R., Ewing J., Taylor P. 2008. Citation statistics, A report from the joint committee on 281 quantitative assessment of research. IMU, ICIAM, IMS). Report from the International Mathematical Union (IMU) in cooperation with the International Council of Industrial and Applied Mathematics (ICIAM) and the Institute of Mathematical Statistics (IMS).

Blackburn JL., Hakel MD. 2006. An examination of sources of peer-review bias. Psychological science 17:378-382. DOI: 10.1111/j.1467-9280.2006.01715.x.

Chapman S., Eisinga A., Hopewell S., Clarke M. 2012. Two-thirds of methodological $\underline{\text { research remained unpublished after presentation at Cochrane Colloquia: an empirical }}$ analysis. Journal of clinical epidemiology 65:493-496. DOI:

10.1016/j.jclinepi.2011.09.009.

Dickersin K., Min YI., Meinert CL. 1992. Factors influencing publication of research results. Follow-up of applications submitted to two institutional review boards. JAMA: the journal of the American Medical Association 267:374-378.

Dressler D., Leswick D. 2015. Canadian Association of Radiologists Annual Scientific Meetings: How Many Abstracts Go on to Publication? Canadian Association of $\underline{\text { Radiologists journal }=\text { Journal l'Association canadienne des radiologistes 66:96-101. }}$ DOI: 10.1016/j.carj.2014.05.005. 
major radiology meetings. Korean journal of radiology: official journal of the Korean

302

Radiological Society 9:303-311. DOI: 10.3348/kjr.2008.9.4.303.

Hoag CC., Elterman DS., Macneily AE. 2006. Abstracts presented at the American

304

Urological Association Annual Meeting: determinants of subsequent peer reviewed

305

publication. The Journal of urology 176:2624-9; discussion 2629. DOI:

306

10.1016/j.juro.2006.08.021.

307

Hopewell S., Loudon K., Clarke MJ., Oxman AD., Dickersin K. 2009. Publication bias in

308

clinical trials due to statistical significance or direction of trial results. Cochrane

309 database of systematic reviews :MR000006. DOI:

310 10.1002/14651858.MR000006.pub3.

Kuczmarski TM., Raja AS., Pallin DJ. 2015. How do Medical Societies Select Science for Conference Presentation? How Should They? The western journal of emergency medicine 16:543-550. DOI: 10.5811/westjem.2015.5.25518.

$314 \quad$ Lokker C., Haynes RB., Chu R., McKibbon KA., Wilczynski NL., Walter SD. 2012. How well are journal and clinical article characteristics associated with the journal impact factor? a retrospective cohort study. Journal of the Medical Library Association: JMLA 100:2833. DOI: $10.3163 / 1536-5050.100 .1 .006$. 2016. Scientific papers presented orally at radiology meetings-trends in subspecialty publication rates and adaptations associated with the highest impact factor journal publications. Quantitative imaging in medicine and surgery 6:462-465. DOI: $\underline{10.21037 / \text { qims.2016.08.10. }}$ 
323 Miguel-Dasit A., Martí-Bonmatí L., Aleixandre R., Sanfeliu P., Bautista D. $2006 a$.

$324 \quad$ Publication of material presented at radiologic meetings: authors' country and 325 international collaboration. Radiology 239:521-528.

326 Miguel-Dasit A., Martí-Bonmatí L., Sanfeliu P., Aleixandre R. 2006b. Scientific papers 327 presented at the European Congress of Radiology 2000: publication rates and 328 characteristics during the period 2000-2004. European radiology 16:445-450. DOI: $329 \quad \underline{10.1007 / s 00330-005-2861-3 .}$

330 Parkar AP., Vanhoenacker FM., Adriaensen van Roij MEAPM. 2013. Publication rate of 331 scientific abstracts presented at ESSR 2008 and 2009. Skeletal radiology 42:561-565. $332 \quad$ DOI: $10.1007 / \mathrm{s} 00256-012-1505-3$.

Rodrigues MA., Tedesco ACB., Nahas FX., Ferreira LM. 2014. Journal impact factor versus $\underline{\text { the evidence level of articles published in plastic surgery journals. Plastic and }}$ reconstructive surgery 133:1502-1507. DOI: 10.1097/PRS.0000000000000214. Scherer RW., Langenberg P., von Elm E. 2007. Full publication of results initially presented in abstracts. Cochrane database of systematic reviews :MR000005. DOI: 10.1002/14651858.MR000005.pub3.

Shelmerdine SC., Lynch JO., Langan D., Arthurs OJ. 2016. Presentation to publication: proportion of abstracts published for ESPR, SPR and IPR. Pediatric radiology 46:1371-1377. DOI: 10.1007/s00247-016-3653-4. 
346 Song F., Loke Y., Hooper L. 2014. Why are medical and health-related studies not being 
Figure 1

Search algorithm to find publications 
First author searches Google then Google Scholar then PubMed using presentation title.

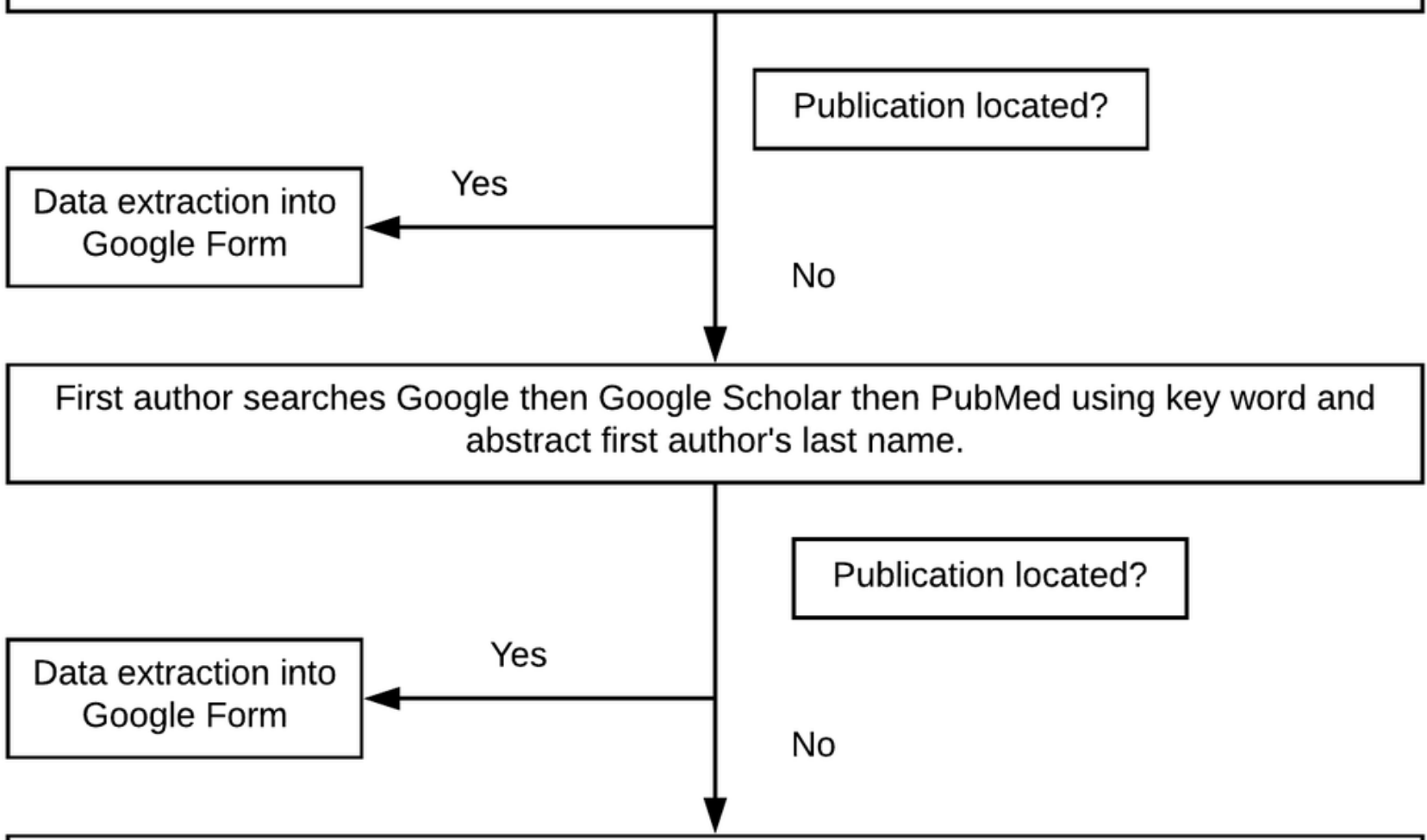

Second author repeats previous two search strategies for non-located abstracts

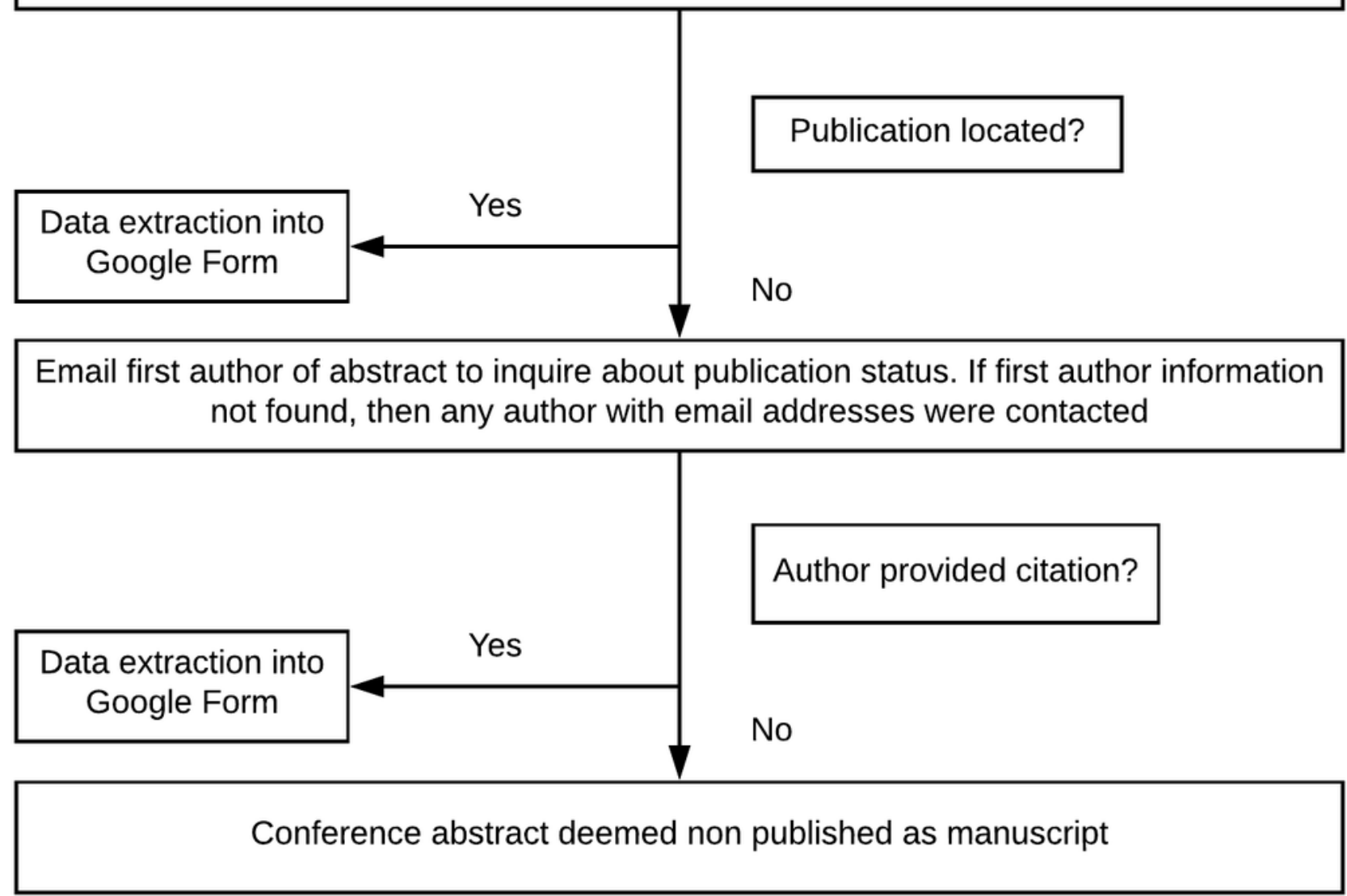


Figure 2

Flowchart for publication analysis 


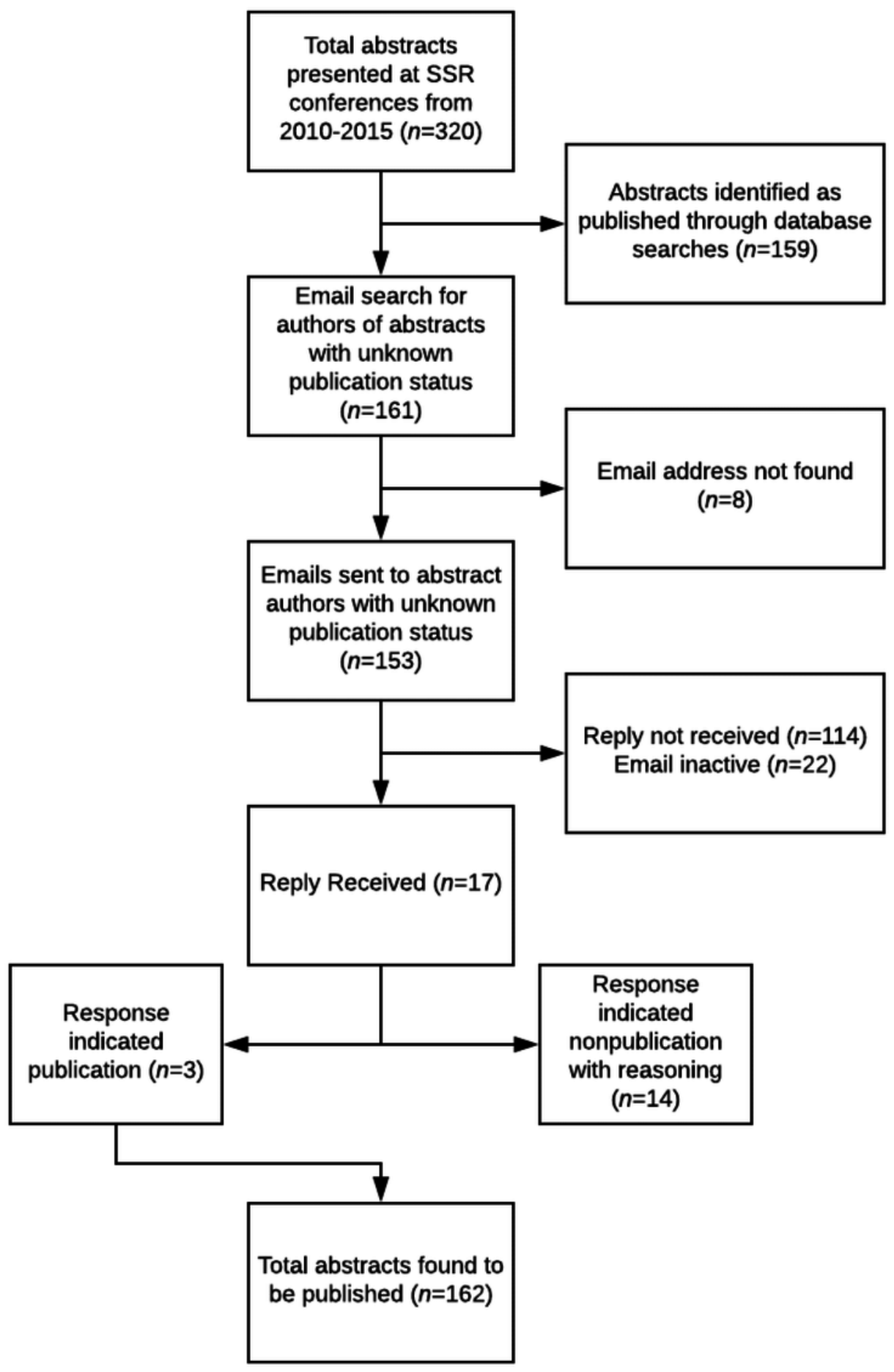


Figure 3

Percent of published studies by conference year 


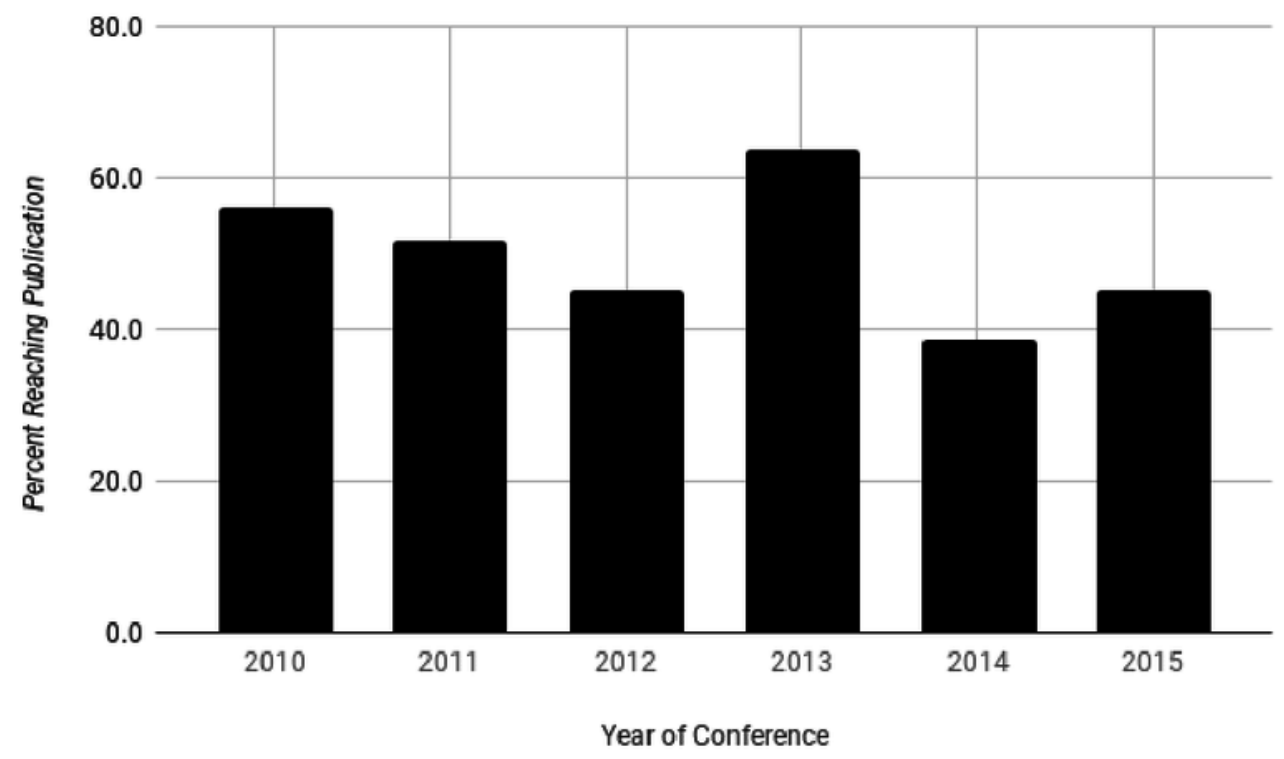




\section{Figure 4}

Kaplan Meier Analysis Excluding Abstracts Published Prior to Conference Date

Studies published prior to the conference date were considered 0's and excluded from the analysis as they are not "at risk" for publication. 


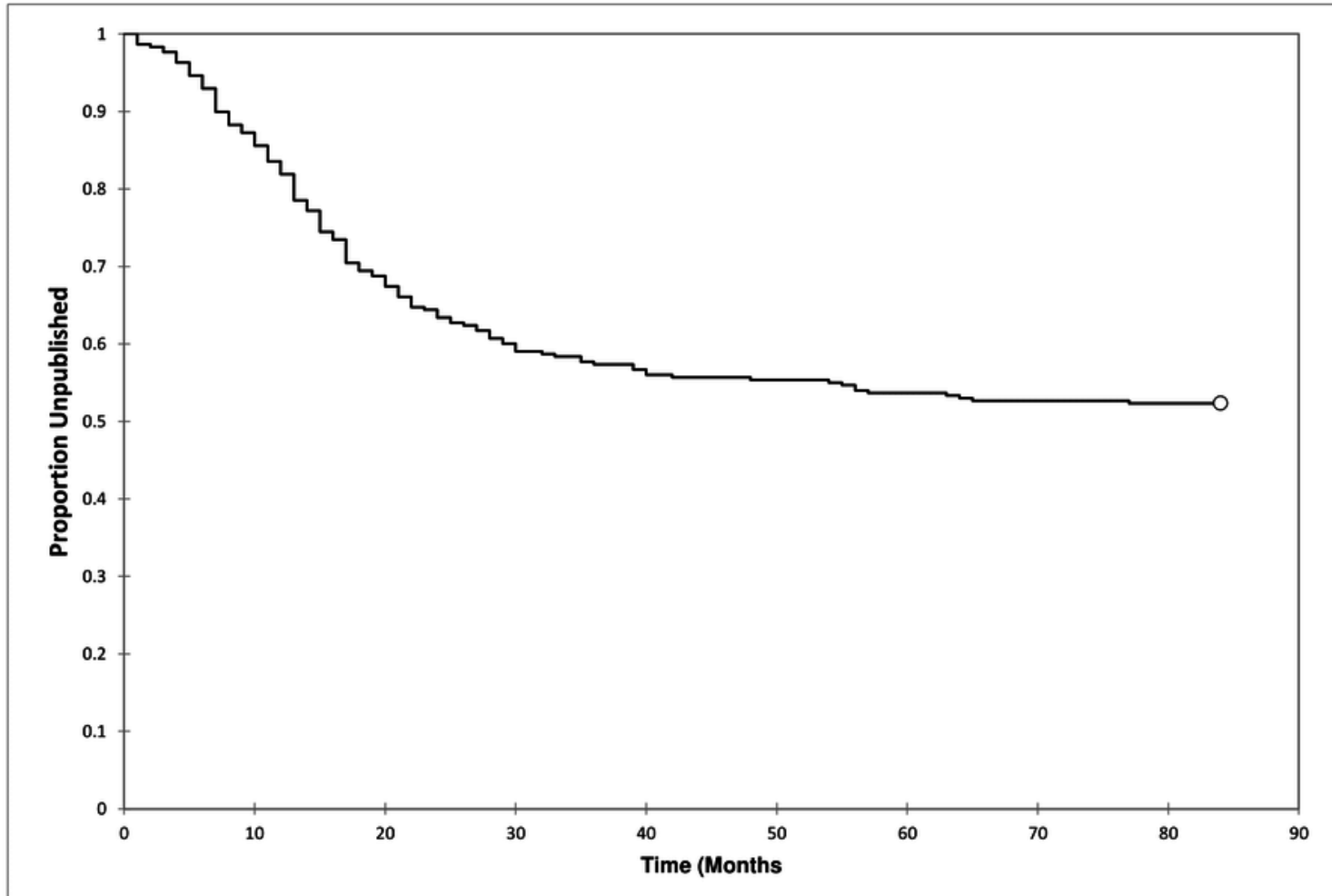

\begin{tabular}{|l|r|r|r|r|r|}
\hline Time (Months) & 0 & 20 & 40 & 60 & 80 \\
\hline $\begin{array}{l}\text { Number } \\
\text { Unpublished }\end{array}$ & 300 & 206 & 171 & 162 & 158 \\
\hline
\end{tabular}




\section{Figure 5}

Journals that Published 3 or more Conference Abstracts

$\mathrm{SR}=$ Skeletal Radiology, AJR = American Journal of Roentgenology, $\mathrm{ER}=$ European

Radiology, JUM = Journal of Ultrasound in Medicine, JMRI = Journal of Magnetic Resonance

Imaging, PP = Pain Physician, JCAT = Journal of Computer Assisted Tomography, EJR = European Journal of Radiology, $C O R R=$ Clinical Orthopaedics and Related Research, ARD = Annals of the Rheumatic Diseases. 


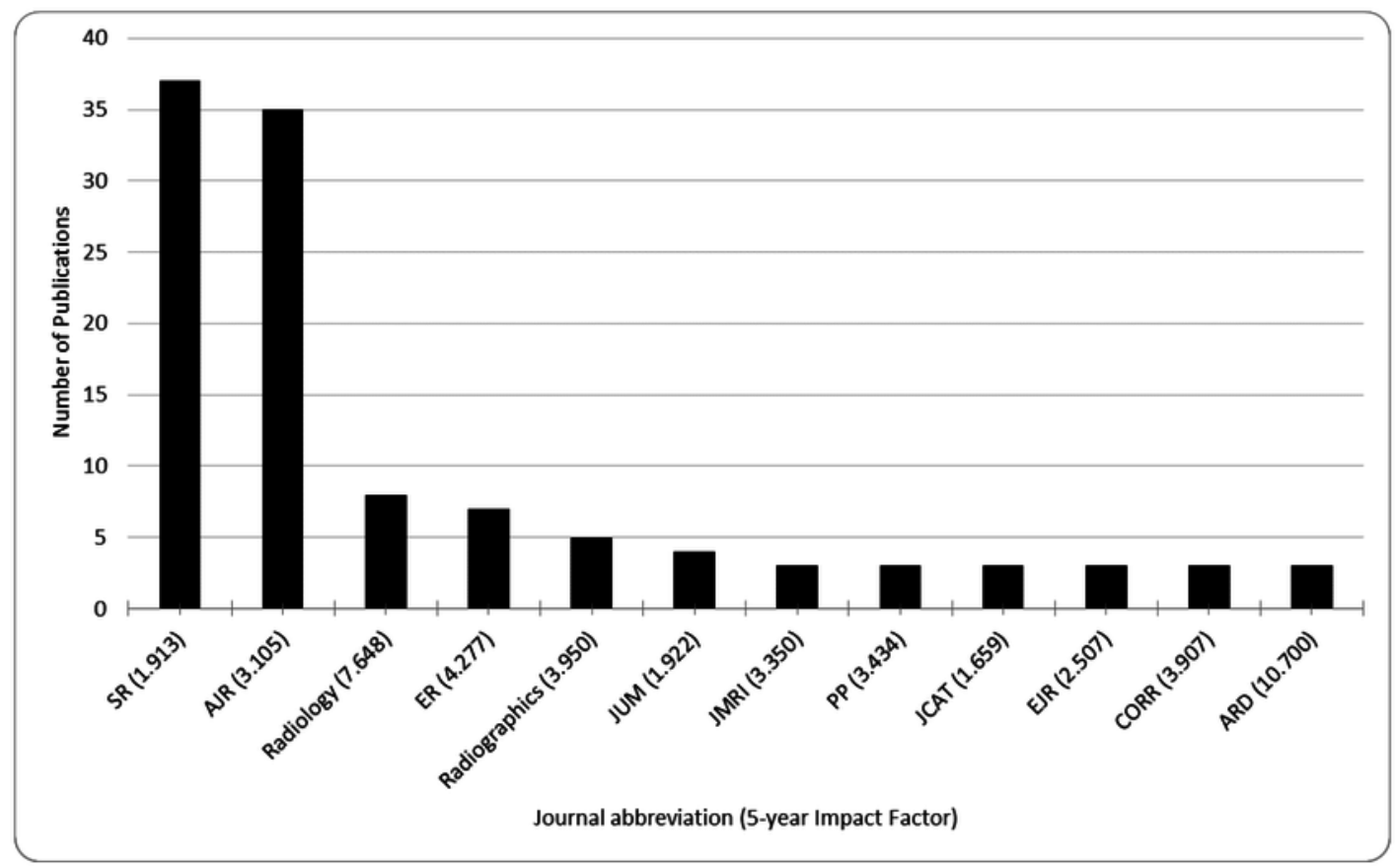

